\title{
Postal Network Reorganization with Implementation of Information Technology
}

Stanislav Levičar* and Andrej Lisec

University of Maribor, Mariborskacesta 7, 3000 Celje, Slovenia

\begin{abstract}
The purpose of this paper is to analyse the possible improvement in logistics solution of Post of Slovenia through reorganisation of the hierarchy of the postal system with the introduction of regional postal centres and parcel post and based on that through improvement of the transportation between the post units and new solutions in informatics. The hypothesis is that by changing the spatial hierarchy and allowing retention and transhipment of parcels among hubs at different levels of economies of distribution costs can be achieved. Additionally, the article proposes the exploration of self-learning automated algorithms to be used in the postal information systems, which could dynamically look for optimums for additional resource management cost reduction opportunities.
\end{abstract}

Keywords: Distribution Hierarchy, Information Technology, Logistics, Postal Logistics Centre, Postal System, Selflearning Algorithms.

\section{Introduction}

This paper deals with the optimization of parcel logistics and with recent information technology. The distribution of parcels is based on a hierarchical postal network. The paper presents process of decision making related to the hierarchical structure inside postal network and to the determination of the optimal routes between postal units at different levels. For the most effective operation of companies that deal with parcel transportation, a decision on the following issues needs to be made:

1. Determination of types of postal units and the hierarchical relationship between them;

2. The location of postal units;

3. The allocation of routes connecting postal units.

New technologies in postal traffic are enabling the modernization of postal systems and logistics in such systems and dictating the use of mathematical opti- mization models in a strategic sense (e.g. taking into account the location and capacity of objects in a postal network) as well as in an operative sense (for example, taking into account the management of 'time windows' as temporal intervals during which a service is completed) (Mason et al., 2007).

When solving optimization problems at the strategic level, the question of the location and capacities of its facilities in postal logistics systems as well as the routes of these spatial networks arise (Simchi-Levi et al., 2003). The same goes for parcel services. In all Posts we need to achieve:

- An effective collection and sorting of parcels;

- A reliable delivery;

- An effective and efficient transport.

The criterion functions require that the total sum of logistic costs in this service of parcels should be minimal, often under certain capacity constraints. 


\section{Literature Review}

In Switzerland as well as in other Central European countries the problem of postal hub location has been studied and presented as vital for efficient postal logistics in quite a few papers. Hierarchical hub location problems are well discussed and reviewed in the theory and practical applications of O'Kelly (1987), Campbell J F (1994), Bruns et al. (2000), Marin (2004) and Ernst et al. (1996).

Successful parcel service application of postal services model is to be found at Swiss post, described in Bruns et al. (2000), which deals with restructuring the parcel service network, and with choosing transhipment points among the nodes in the network. They used a discrete facility location model. Hub transportation network for parcel delivery service in Austria is described in paper of Wasner et al. (2004), where an integrated three level multi-depot hub-location vehicle routing model for network planning of parcel service in Austria is mentioned. Wasner et al. (2004) described in their paper why the optimal design of depot and hub transportation networks for parcel service providers makes it necessary to develop a generalized hub location and vehicle routing model. Using a hierarchical model, they searched for an optimal number of depots among potential locations for the Austrian parcel service (searching the shortest sum of lines of all cycles in hierarchical structures of the Austrian postal system). Ernst et al., (1996) provided a numerical example on the Australian Post data set for the incapacitated single allocation $\mathrm{p}-$ hub median problem for only 25 nodes (reduction from 200 nodes). They consider uncapacitated multiple and single allocation p-hub median problem.

Ebery et al. (2000) described the capacitated multiple allocation as the hub location problem and developed a new mixed integer linear programming formulation for the problem. They constructed an efficient heuristic algorithm, using shortest paths. This paper is based on a revised version of the Ebery et al.'s model (2000) that deals with the flow of shipments from the initial phase through the centre/or between centres to the end consumer. The original model has been expanded for the needs of optimal parcel logistics with parcel distribution at various levels. Here, the separation of dispatches at various levels with the aim of retaining parcels for one's own area is considered, although this was not the case in the model on which this study is based. Therefore our research question is what is the impact of the number of hierarchical levels, which determines the possibilities for separation of dispatches and transhipment at various levels, on the efficiency of the transport network? Our hypothesis is that by changing the spatial hierarchy and allowing retention and transhipment of parcels among hubs at different levels economies of transport cost can be achieved.

To give answer to this research question the basic concept of Ebery et al.'s model (2000) is used to evaluate a four-level distribution of parcel delivery. Our paper aims to upgrade their approach to evaluate the flows between Postal Logistics Centres - (PLC) and Regional Parcel Centres - RPC patronizing the given set of Parcel Posts - PP. In our approach, the number of chosen PPs and RPCs has its upper limit and is chosen among the existing posts as the potential central places in spatial hierarchy. It depends on the volume of parcel flow coming from or going to a certain RPC per day and can increase by increasing volume of parcel flow.

\section{Problem Identification}

The purpose of this paper is to analyse the possible improvement in logistics solution of Post of Slovenia through reorganization of the hierarchy of the postal system with the introduction of regional postal centres and parcel post and based on that through improvement of the transportation between the post units. The economy of scale achieved by the 4-level structuring of hubs in central places of different levels as nodes is analysed for the case of Post of Slovenia delivery service with the expansion of the results of Ebery et al. (2000). With the introduction of regional parcel centres and parcel posts the current two-level parcel distribution (postal logistics centres and posts) is reorganized into a four-level one, with the objective to overcome 
problems that arise at the postal logistics centres and to reduce transport costs. The function of the postal logistics centre remains the same. The postal logistics centre continues collecting, processing and dispatching parcels. The only difference is that the trucks collect and deliver parcels to a greater extent from and to the regional parcel centres. Up to now they have only collected and delivered parcels from and to smaller post offices.

The article critically examines the idea of introducing regional parcel centres and parcel posts along with the optimal organization of parcel distribution. A postal logistics centre is a collection centre collecting parcels from regional parcel centres and parcel posts so the economic efficiency of the entire transportation system can be achieved. The postal logistics centre encompasses the volume of parcels from specific points of demand. All points of demand form part of the regional parcel centre as well as the postal logistics centre. The key to the quality and rational distribution of parcels includes an optimal location of postal logistics centres, regional parcel centres and parcel posts. The hierarchical transport network has to be built, connecting Posts, Parcel Posts, Regional Parcel Centres and Postal Logistics Centres, where the total costs of daily transhipment of all parcels would be minimal. The number of vehicles used for transportation of parcels has to be determined; the same goes for the timetable for the collection and delivery of mail.

\section{The Current Logistics of the Post of Slovenia}

Post offices accept mail throughout the day. Mail is taken from there to one of the two postal logistics centres in Slovenia using designated trucks at least once a day. Today there are two postal logistics centres in Slovenia, one in Ljubljana and the other in Maribor. The two logistics centres in Ljubljana and Maribor process and redirect the mail. An exchange of mail between the two centres also takes place at night. In the morning mail is dispatched from both logistics cen- tres to individual post offices. Trucks from bigger post offices deliver mail to some smaller ones. Postmen then deliver the mail to their correct addressees.

Each shipment is transported by a vehicle at least two times: from the collection post to the PLC and from the PLC to the addressee. Hence, mail is transported on various routes:

- from the sender to the post office;

- from the post office to the PLC (sending mail from the post office to the PLC);

- from the PLC (Ljubljana or Maribor) to another PLC (Maribor or Ljubljana);

- from the PLC to a post office (arrival of mail from the PLC to the post office);

- from the post office to another post office (for smaller post offices a small delivery vehicle delivers mail to the bigger ones, smaller remote posts collect mail in the early hours or deliver it to the bigger post offices in the afternoons or evenings);

- from the post office to the addressee; and

- from abroad to the PLC and vice versa.

The existing logistics concept is based on the concentration of all collected parcels at both postal logistics centres, in Ljubljana and Maribor, where they are directed and sent to the relevant post offices along with the rest of the mail. These means that all of the parcels collected in Slovenia are directed either to Ljubljana or/ and Maribor. Parcels that are collected at postal units in Maribor, Celje and MurskaSobota are brought to the PLC in Maribor, parcels that are collected at the postal units in Ljubljana, Kranj, Nova Gorica, Koper and Novo mesto are brought to the PLC Ljubljana. The latter case will be introduced and studied in more detail. The highest number of shipments (some 70 percent) is collected and transported from the Postal Logistics Centre in Ljubljana on a daily basis. Data were only collected for this area covering two-thirds of Slovenia.

Previous case studies by Lisec et al. (2005) and Lisec, Rusjan (2011) have proven that dividing Slovenia into 


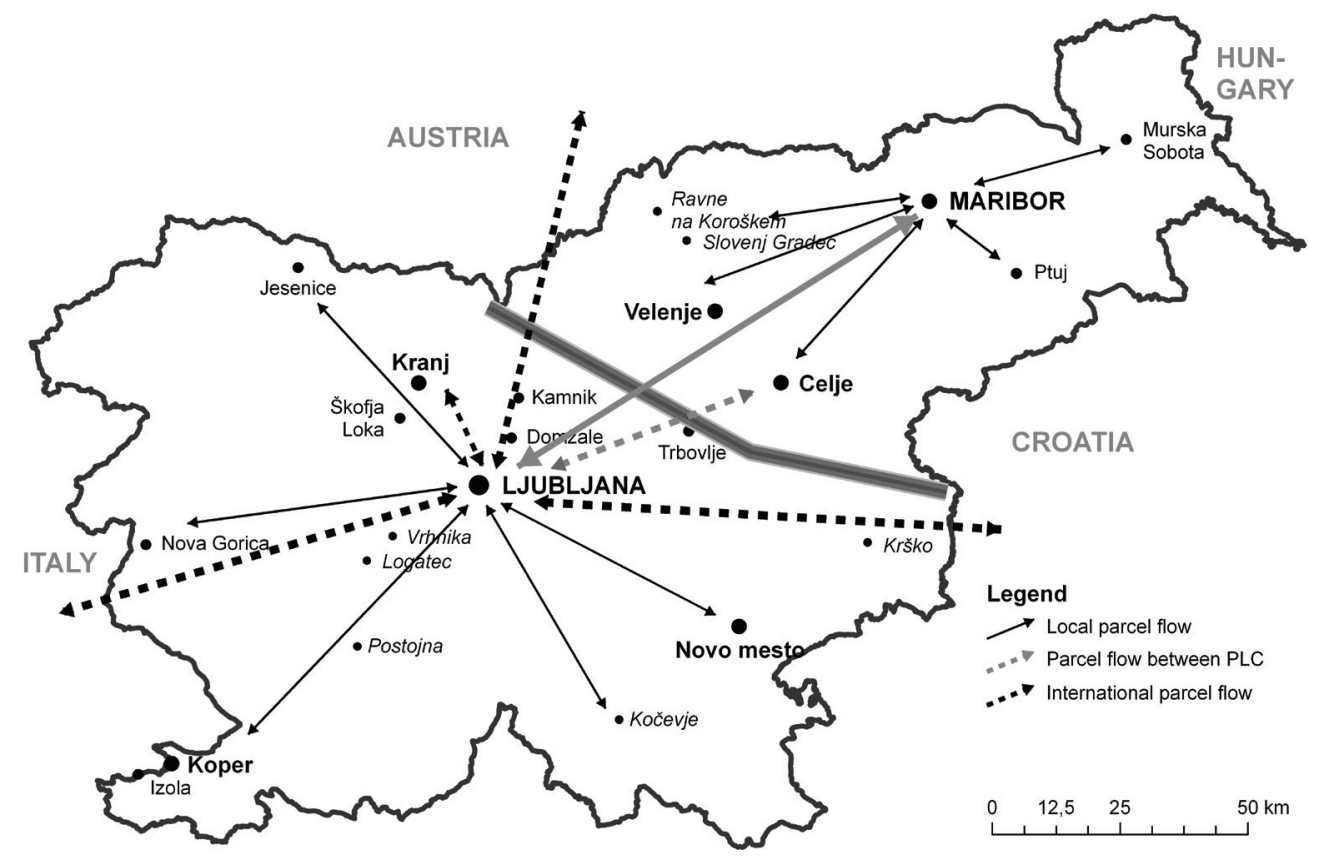

Figure 1. The parcel flows in Slovenia.

two PLC areas is still adequate considering the volume of mail and parcel deliveries, however, the increased volume of services already calls for certain changes due to predictions of greater mail flows in the next few years.

The current logistics concept suggests that the two postal logistics centres are facing the following problems:

- an insufficient number of available vehicles at peak times;

- an insufficient number of available drivers at peak times;

- the consistency in assurance of delivery deadlines D +1 (if it is collected today that means it is delivered tomorrow), especially for big-sized parcels;

- inadequate space (for manipulations and inter-phase warehousing) in a PLC; and

- the inadequate spatial capacity of vehicles at the first and the second transport level.

\section{New Concept of the Postal Logistics System}

In the present mode of postal logistic system all the connections between individual postal units are made through PLC Ljubljana and/or Maribor. However data on the number of parcels collected in a study, done in December 2007, of the Nova Gorica and Koper postal units have shown that it is reasonable to connect the two locations directly. This is especially effective when the daily volume of collected or dispatched parcels between both regional centres increases (Lisec et al., 2005). It may be assumed that direct connections might also be reasonable between other bigger towns in Slovenia (Lisec et al., 2005).

Therefore, the current concept of logistics system of the Post of Slovenia is suggested to be altered on the basis of the revised and expanded model introduced by Ebery et al., (2000), with the introduction of a four- 
level transport of parcels (Lisec, Rusjan, 2011), based on the current postal logistics centres and introduction of new regional parcel centres and parcel posts.

The Posts on the level of local communities, patronizing a certain area, have to be assigned to the proper Parcel Post.

Regional parcel centres process mail locally and for the areas of other regional parcel centres in the future. Regional parcel centres as such deal with the collection and delivery of mail. Their task is to collect and deliver mail to clients in the wide area of the city and direct it to the parcel posts (in addition to delivery and dispatch from the postal logistics centre). At the same time the regional parcel centres collect, dispatch and deliver parcels of bigger dimensions in the area of regional parcel centres. In the future, the increased volume of parcels will enable the regional parcel centres to direct parcels between them as the transporting of all parcels to the existing postal logistics centres will become questionable due to the huge volume and dimensions and will represent an extremely complicated logistics process. In the event of continuing growth in the volume of parcels the regional parcel centres will need transport conveyor belts or smaller appliances for the separation process, which at this point will not be taken into account.

The justification of introducing regional parcel centres with the aim of separating and excluding parcels for the local area will be studied due to the increased volume of parcels and especially due to rationalization of the system, for the two logistics centres have problems ensuring the direction of all parcels in one night. Problems that occur in the existing mode of transporting shipments relate to the organization of transportation, while the result of these problems is that parcels are retained in PLCs for up to two days. This does not comply with the prescribed time window D+1 mentioned before.

At the same time, with the allocation of the regional parcel centres, the allocation of parcel posts that cover smaller areas within the wider areas of the regional par- cel centres has to be taken into account. Geographically, parcel posts usually belong to the area of the regional parcel centre. The role of parcel post is the collection and delivery of parcels in the local area and associated post offices as well as collection and delivery on the spot. Parcel posts redirect mail and parcels to smaller post offices (some bigger post offices already practice this).

Using the basic concept of four level hierarchy of postal system and applying it to the case of the Post of Slovenia, the usefulness of allocating regional parcel centres at different locations (in addition to the two existing postal logistics centres in Ljubljana and in Maribor) is analysed. We analyse their economic legitimacy and the appropriateness of their location for covering the Ljubljana area. The hierarchy of the area covered by the PLC Ljubljana shall therefore be as follows:

- one postal logistics centre; and

- at most five regional parcel centres (for at least thirty post offices). Each centre would cater for 3-12 parcel posts depending on the number of collected and delivered parcels. In their local area, parcel post offices cater for 3-20 post offices.

These requirements are only fulfilled by the following post offices: the Postal Logistics Centre Ljubljana that also has the function of the regional parcel centre, the parcel post and the post office, post $4101 \mathrm{Kranj}$, 6104 Koper, 8101 Novo mesto, and 5102 Nova Gorica which also operate as both parcel post and regular post offices.

Next, the cost limits for the introduction of all four regional parcel centres are calculated. To this end, the logistics cost structure has to be addressed. Logistics costs may be divided into fixed and variable ones. Fixed costs are made up of investment costs and operational costs. The former are calculated on the day of operation, while the latter do not depend on the parcel quantity processed at a particular post office. Variable costs are costs that are proportional to the quantity of mail shipments. In order to justify the allocation 
of regional parcel centres, the model of Bruns et al., (2000) was used.

The time window is represented by $\left[e_{i}, l_{i}\right]$ and, to each arc $(i, j) \in \mathrm{A}$, there is an associated cost $\left(c_{i j}\right)$ and travel time $\left(t_{i j}\right)$. The travel time matrix is symmetric $\left(t_{i j}=t_{j i}\right)$ and with strictly positive entries $\left(t_{i j}\right.$ $>0$ ). $e_{i}$ is the earliest time for delivery (or collection) and $l_{i}$ the latest time for delivery (or collection).

The following formalization has been used: subject to:

$$
\min \sum_{(i, j) \in A} c_{i j} x_{i j}
$$

subject to:

$$
\begin{aligned}
& \sum_{J \in N} x_{i j}=1 \quad \forall i \in N \\
& \sum_{J \in N} x_{j i}=1 \quad \forall i \in N
\end{aligned}
$$

if $x_{i j}=1$ then $p_{i}+t_{i j} \leq p_{j} \quad \forall(i, j) \in A$

$e_{i} \leq p_{i} \leq l_{i} \quad \forall i \in N$

$x_{i j} \in\{0,1\} \quad \forall(i, j) \in A$

The variable $x_{i j}=1$ means that $\operatorname{arc}(i, j)$ is used, otherwise. The variable $x_{i j}=0$. The variable $p_{i}$ represents the departure time from node $v_{i}$.

Equation 1 and 2 define an assignment problem of dimension $(n+1)$. Equation 3 and 4 represent the time window constraints.

Regional parcel centres are excluded sequentially when altering additional fixed costs in steps of the size of one monetary unit (Rupee 95.53). Additional fixed costs are expressed as the equivalent of the travel costs per kilometre with a truck in both directions. The costs also include the appropriate salary part of the driver. 2961.47 worth of fixed costs was calculated (31 monetary units of fixed costs) as a cut-off point in justification of the transformation of a regular post office into a Regional Parcel Centre Kranj. On the contrary, additional fixed costs of 3057.00 per day, (32 monetary units of fixed costs) make the allocation of the RPC Kranj unjustified.

We also calculated the maximum amount of additional fixed costs incurred as criteria for the justification of regional parcel centres for other ordinary post offices (Koper, Novo mesto, Nova Gorica). The results are the following: up to 6496.13 (68 monetary units) of additional fixed costs per day justifies the allocation of the RPC Koper, 6591.66 (69 monetary units) of additional fixed costs per day justifies the allocation of the RPC Novo mesto and 15093.11 (158 monetary units) of additional fixed costs justifies the RPC Nova Gorica. If higher additional fixed costs are identified for the regional parcel centres then a four-level service is not justified (when calculating the actual additional fixed costs only those costs should be taken into account that are incurred through additional building construction and equipment, reduced by the reducibility of such costs at the higher level).

We calculated the marginal cost acceptable to introduce the specific RPC in order to take these cost into account as additional cost later, when we calculate transportation cost under the new four level hierarchical postal systems.

The optimization of parcel shipment flows to PLC Ljubljana, with the new hierarchical structure of the postal network is described in paper Multi-level postal network for improved efficiency: A case study of Slovenian post (Lisec, Rusjan, 2011).

The structure of vehicles of various capacities that the Post of Slovenia has at its disposal was also taken into account in the algorithm (Zmazek, Zerovnik, 2005). First, routes at the highest level (PLC-PLC) were determined, followed by RPC-PLC, the PP-RPC level and last but not least the post office-PP level. In the calculation the time windows were also considered. 


\section{Calculation of Transportation Costs for PLC Ljubljana with the New Timetable}

Total costs include the transportation of parcels (vehicle and driver costs), but not the costs of the collection, delivery and redirection of parcels at both logistics centres or the administrative costs of employees which are constant regardless of the way the flows are located in the network.

The new organization of transporting shipments requires 26 trucks (excluding broken down vehicles). The total number of kilometres covered by trucks in the new system is 6263. Some drivers of middle-sized vehicles or light delivery vans can operate on several routes per day. Two additional trucks are taken into account as a backup due to potential breakdowns (they were also taken into account in the current mode of transport). For this reason the total number of trucks is 28 .

The routes between individual post offices, parcel posts, regional parcel centres and postal logistics centres ought to be operated by 29 middle-sized delivery vans and 57 light delivery vans. Middle-sized delivery vans should cover 424 kilometres in the PLC, RPC and PP areas, and 2298 kilometres within the parcel post office area. The total number of kilometres covered by middle-sized delivery vans is thus 2722 kilometres. Light delivery vans would cover 130 kilometres in the PLC, RPC and PP area and 3652 kilometres within the parcel post area (3413 kilometres by light delivery vans and 239 kilometres by busses and trains). The number of vehicles would remain the same, that is, 57 light delivery vehicles from the existing mode of transport.

Table 1. Cost analysis of the new transportation mode

\begin{tabular}{lllllll}
\hline Vehicle & Fixed costs of a vehicle per day & Fixed costs of a driver per day & Variable costs & No. of vehicles & No. of drivers & No. of km per day \\
\hline TV & 5466.42 & 6517.23 & $16.91 € / k m$ & 28 & 52 & 6263 \\
SDV & 1255.43 & 6517.23 & $6.76 € / \mathrm{km}$ & 29 & 22 & 2722 \\
LDV & 851.33 & 6517.23 & $6.76 € / \mathrm{km}$ & 57 & 15 & 3782 \\
\multicolumn{2}{l}{ Total number of $\mathbf{k m}$ per day } & & & & $\mathbf{1 2 7 6 7}$ \\
\hline
\end{tabular}

The total number of kilometres covered is thus 3782 kilometres. Within the parcel post area several vehicles may be used in order to comply with the particular time window either in the morning or in the afternoon. At the lowest level, there is no danger of exceeding the capacity limit of certain vehicles. If there are several routes within the parcel post, the routes are designed according to the principle of rounds with the routes not overlapping each other. This, however, does not affect the routes operated in the afternoons as post offices close at different hours.

The total number of kilometres covered per day between postal logistics centres, regional parcel centres, parcel posts and post offices is 12767 . This reduces the total length by 6250 kilometres.

The number of vehicles assigned to the regional parcel centres (so far they were located at the PLC Ljubljana) is determined in relation to the optimal solution. By rearranging trucks for RPC for the collection and delivery of parcels, we expand the existing system of parcel logistics so that the Post of Slovenia can cope with the increased volume of parcels both at present and in the future. If this is the case, the PLC Ljubljana would not need any additional trucks for parcel dispatch.

The costs of the new transport mode are 1026212.76€ per day (USD 1385387.23). If additional costs of the allocation of the regional parcel centres totalling $31142.37 €$ (USD 42042.20) are added to the costs of the optimal mode of transport created through the introduction of the retention and separation of parcels, we get $1057355.13 €$ per day (USD 1427429.43). In comparing the costs of the present and new modes of transport (separation of parcels at post offices and parcel posts) an expansion of the workforce is not taken into account as our assumption is that the workers are 
reallocated to regional parcel centres from the postal logistics centre, whereby the additional fixed costs of regional parcel allocation per day are taken into account.

It is clear that by introducing the new mode of parcel distribution, and by introducing the regional parcel centres, the costs of the parcel distribution would fall by 265803.67€ per day, USD 358834.96 (79741100.18€ per year, USD107650485.20). Distribution costs are, as a result, reduced by more than 20 percent compared to the current mode of transport.

\section{Postal Networks Empowered by Information Technology}

Besides systematically reorganization of the postal network, additional sources of cost reduction can be developed by implementation of recent information technology solutions that enhance the predictability of the processes is recommended. A more detailed information about the flows that have to be managed, facilitates further optimization of the usage of available infrastructure of the postal network, since the deviations can be detected in its earlier phases, thus enabling the organization to adapt and deploy the resources (i.e.. trucks, workforce, etc.) according to them (Gupta and Krajewski, 2003).

Additionally, useful information can be gained by tracking not only the target destinations of addressees (Mercier et al., 2008), but also the addresses of the senders to gain the insight into the patterns that might emerge by combining such data and dissecting them by managed or - to a certain degree - the self-learning algorithms. Their inputs should not be limited only to the information about post and parcels, but should include also the various data that can be identified as being relevant to a certain degree to the quantity or type of those postal flows.

The initiatives that are being spread currently are based on introduction of RFID applications in the postal ser- vices, which enable easier and more real-time access to the location of the shipments internally (while inside the postal company), and the even further enriched data about the parcels by GPS mapping. Combined they offer much more granulated and precise information about the current location, the usual path and the detailed information about the bottlenecks that emerge along the itinerary. The data that is also available freely - at least to a certain degree - is the address of the sender, which was usually not read, even if it was marked on the envelope or the outside package of the parcel.

By evaluating such data the key sources of the bottlenecks can be identified (Carmi, 1999). Due to the effect of compounding that is known to queuing theory, even the slight deficiency of one phase in the process amounts to substantial delays in the delivery times of the entire batch of post. Such events can be avoided by early warnings that would cause temporary allocation of resources or the dynamic diversion of the incoming post to another facility that might have surplus capacities and would therefore handle the post much faster and efficiently even though they are not the most optimal in normal situations.

Another possibility that should be evaluated further is the possibility of early warning systems to the general users, and especially to those that are - when combined - causing the deviations in the postal processes, with the aim to encourage them to rearrange their planed shipping's. Many times they might be ambivalent of the shipping dynamics and might be willing to adjust their sending scheme without any further ado.

More visionary cases would include even tight integration between postal companies and their clients, which would enable them to use the postal infrastructure in new unprecedented ways, like for transferring of printing the post (in the name of client) to a place that is closer to its recipients. Such approach could substantially decrease costs and increase the flexibility and the speed of the postal services while extending the time 
clients can make adjustments to the content of their post even further.

Although the advantages of those new postal processes are clear, there are two main obstacles that are preventing their realization and implementation. Firstly, the information that can be gathered about parcels and other post is usually incomplete to a degree that may become unusable for developing new strategies and concepts of the post organization. The cost of the modifications of the existing organization might then prove to be greater than the potential benefits (since they would be the result of the reorganization that would have to take into account limitations that are stemming from not having the exact information about important part of the parcels and other post).

Secondly, the vast and significant improvements are possible especially in the area of the external information systems integration. Other government agencies, institutions and companies (in the first place the larger users of the post services) could provide inputs to the postal information systems which could in response rearrange and displace the available resources according to the expected distribution of work in the different areas.

This second possibility can also be rendered invalid if the collected postal data is not encompassing the representative information of the population of parcels and other post. The high level of fragmentation and incomplete information about the senders makes it more difficult to predict those postal flows in advance. Therefore, the information systems that would be put in place should not only rely on the input data, but should reprocess those data extensively with the aim of finding the patterns that may become predictable in advance and could only this way significantly help forecasting the desired optimums (Zhang and Meng, 2007). Such systems can be made even more useful with the extended scope of input data, that would include not only the postal related information, but from many other factors that consequentially and finally do have the impact on the postal flows. To make use of such systems they would necessarily have to rely on heavy real-time computing and self-learning algorithms. One of the potential challenges when introducing such systems is the very probable of the short term degradation of the already established optimums, since the system is only trying to begin looking for other solutions and by evaluating the provided data it could propose the reorganization that might not include all the factors and their proper values to be able to correctly predict its outcome. Therefore the certain level of vigilance has to be present especially at the introduction to prevent possible excess solutions. After initial period when the system would become more reliable, such tight monitoring would not be necessary but the continued improvements would still require certain level of engagement on the constant basis.

\section{Conclusion}

Having studied the four-level model, we may conclude that it would be sensible to introduce a new way of distributing parcels by creating regional parcel centres and parcel posts involving the separation process of parcels for the local area and directing mail shipments to freight parcels on scheduled routes. In this way the transport costs (logistics) would be reduced daily by 20 percent. The possibilities that could enable additional cost reduction could be realized by usage of the advanced information systems.

\section{References}

Bruns, A., Klose, A., \& Stahly, P. (2000). Restructuring of swiss parcel delivery services. Operations ResearchSpectrum, 22(2), 285-302.

Campbel, J. F. (1994). Integer programming formulations of discrete hub location problems. European Journal of Operational Research, 72, 387-405.

Carmi, N., \& Ronen, B. (1999). An empirical application of the information-structures model: The postal authority case. European Journal of Operational Research, 92(3), 615-627.

Ebery, J., Krishnamoorthy, M., Ernst, A., \& Boland, N. (2000). The capacitated multiple allocation hub location problem: Formulations and algorithms. European Journal of Operational Research, 120(3): 614-631. 
Ernst, A. T., \& Krishnamoorthy, M. (1996). Efficient algorithms for the uncapacitated single allocation $\mathrm{p}-\mathrm{hub}$ median problem. Location Science, 4(3), 139-154.

Gupta, J. N. D., \& Krajewski, L. J. (2003). Operations research in postal services - a survey. Computers \& Operations Research, 4(4), 235-246.

Lisec, A., Ferbar, L., \& Bogataj, M. (2005). Regional aspects of parcel delivery optimisation of the Post of Slovenia: An application for the Novo mesto business unit. Economic and Business Review, 7, 35-55.

Lisec, A., \& Rusjan, B. (2011). Multi-level postal network for improved efficiency. African Journal of Business Management, 5(29), 11676-11685

Marin, A. (2004). Formulating and solving splittable capacitated multiple allocation hub location problems. Computers and Operations Research, 32(12), 30933109.

Mason, R., Lalwani, C., \& Boughton, R. (2007). Combining vertical and horizontal collaboration for transport optimization. Supply Chain Management, 12(3), 188-189.

Mercier, D., Cron, G., Denœuxa, T., \& Masson, M-H. (2008). Decision fusion for postal address recognition using belief functions. Expert Systems with Applications, 36(3), 5643-5653.
O'Kelly, M. E. (1987). A quadratic integer program for the location of interacting hub facilities. European Journal of Operational Research, 32, 393-404.

Simchi-Levi, D., Kaminsky, P., Simchi-Levi, E. (2003). Designing and Managing the Supply Chain (2nd ed.). McGraw-Hill, New York, NY.

Wasner, M., \& Zapfel, G. (2004). An integrated multi - depot hub - location vehicle routing model for network planning of parcel service. Production Economics, 90(3), 403-419.

Zhang, M., \& Meng, L. (2007). An iterative road-matching approach for the integration of postal data. Computers, Environment and Urban Systems, 31(5), 597-615.

\section{About the Author(s)}

Stanislav Levičar is a Teaching Assistant and $\mathrm{PhD}$ student at the Faculty of Logistics, University of Maribor, and can be contacted at: stanko.levicar@gmail.com.

Andrej Lisec(corresponding author)is an Associate Professor at the Faculty of Logistics, University of Maribor, and can be contacted at andrej.lisec@fl.unimb.si. 